\title{
Method to correct the distortion caused by amplified stimulated emission as motivated by LIF-based flow diagnostics
}

\author{
Xuesong Li, ${ }^{1}$ Yan Zhao, ${ }^{2}$ and Lin $\mathrm{Ma}^{1, *}$ \\ ${ }^{1}$ Department of Aerospace and Ocean Engineering, Virginia Tech, Blacksburg, Virginia 24061, USA \\ ${ }^{2}$ Air Products \& Chemicals Inc., Allentown, Pennsylvania 18195, USA \\ ${ }^{*}$ Corresponding author: Linma@vt.edu
}

Received 9 November 2011; accepted 19 January 2012;

posted 31 January 2012 (Doc. ID 157927); published 18 April 2012

\begin{abstract}
Amplified stimulated emission (ASE) represents a significant issue in two-photon laser-induced fluorescence (TPLIF). The ASE effects are nonlinear and nonlocal, i.e., the ASE effects distort the LIF signal nonlinearly, and the distortion at one location depends on conditions at other locations. In this sense, the ASE effects pose a greater challenge to quantitative TPLIF than quenching and ionization. This work therefore seeks a method to correct such distortion. The method uses two LIF measurements, one with low signal-to-noise ratio (SNR) and negligible ASE distortion and another with high SNR but significant distortion, to generate a faithful measurement with high SNR. Extensive simulations were performed to evaluate the performance of this method for practical applications. (C) 2012 Optical Society of America OCIS codes: $\quad 300.2530,300.6420,120.1740$.
\end{abstract}

\section{Introduction}

Among the numerous laser diagnostics developed, techniques based on laser-induced fluorescence (LIF) offer several key virtues, including species selectivity, strong signal to enable two-dimensional imaging measurements of minor species, and relatively simple instrumentation [1,2]. Due to these virtues, LIFbased diagnostics have been extensively applied in a wide range of areas. However, many species of great interest to combustion and plasma research have LIF transitions in the vacuum-ultraviolet (VUV) spectral range [3]. These species include most of the light atoms (e.g., hydrogen, carbon, oxygen, and fluorine) $[\underline{3}, \underline{4}]$, the noble gases (e.g., krypton and xenon) $[5,6]$, and some molecular species (e.g., carbon monoxide and ammonia) [7-9]. To circumvent the experimental difficulty encountered in the VUV range, multiphoton LIF techniques were developed to excite

$1559-128 \mathrm{X} / 12 / 122107-11 \$ 15.00 / 0$

(C) 2012 Optical Society of America the target species via the absorption of two photons (i.e., two-photon LIF, TPLIF). Besides avoiding the experimental difficulty, TPLIF also enables a more comfortable spectral separation between the excitation wavelength (usually in the UV range) and the fluorescence wavelength (usually in the nearinfrared region) in comparison to one-photon LIF.

However, the two-photon process also creates complications [3]. One key issue involves the small twophoton cross section area, which motivates the use of laser pulse with high radiance for excitation to enhance signal and to enable two-dimensional (2D) measurements. The use of excitation pulse with high radiance can trigger several side effects, with photochemistry [10] and amplified stimulated emission (ASE) [11] being the two most notable ones. This work focuses on the ASE effect. As illustrated in Fig. 1(a), the laser field created by the excitation pulse, when strong enough, causes a nonnegligible population inversion between the excited state 3 (with a population of $n_{3}$, and the same notation is used hereafter) and state 2 . When such population 
inversion occurs, a LIF photon, as it propagates through the media, can stimulate a transition from the excited state to a state with lower energy and generate another photon. The photon generated subsequently stimulates other transitions and generates additional photons. When such a process is sustained by the population inversion, ASE, an amplification of the stimulated photons, occurs. When the ASE process occurs, the target species to be measured essentially behaves as an active gain media.

The ASE process represents an opportunity for a new diagnostic tool and at the same time complicates the TPLIF diagnostic. The ASE signal can be a directional, laser-like signal, and it is therefore attractive for diagnostic purposes $[11,12]$. However, the ASE signal depends nonlinearly on many factors, including the number density of the target species (i.e., $n_{1}$ ), the excitation radiance, and the temporal behavior of the excitation pulse. As a result, it is difficult to quantify the ASE signal. Furthermore, the ASE process depopulates the excited state $\left(n_{3}\right)$, causing a nonlinear dependence of the LIF signal upon the aforementioned factors and complicating the interpretation of the LIF signal $[6,13]$.

There is a research need to model and quantitatively understand the ASE effect so that its use as a diagnostic tool can be quantified and its effects on the LIF signal can be corrected. Such consideration has motivated a large amount of modeling efforts around the ASE effect. Models based on the rateequation approximation represented a significant portion of past work due to their simplicity [14-21]. Rigorous models included those based on the density matrix formulation [22,23] and the Maxwell-Bloch equations [24]. These models have thus far been mostly limited to relatively simple one-dimensional (1D) applications, and extension to realistic scenarios is not trivial. Recently, our group developed a model based on the Monte Carlo (MC) method to simulate the ASE effect [25]. The MC method was validated against other models in $1 \mathrm{D}$ and previous experimental data. The results obtained in [25] demonstrated that the MC method offers several distinct advantages, including simplicity in implementation and the capability to model realistic conditions (e.g., temporal and spatial profile of excitation pulse, complicated three-dimensional (3D) geometry, and nonideal optical components).

Based on these previous efforts, this current work applied the MC model to examine the ASE effect and its influence on the LIF signal with the goal of developing a method to quantitatively interpret TPLIF measurements. The major contribution of this work is the numerical demonstration and evaluation of a method that can quantify the LIF signal in the presence of ASE effects. The method involves measuring the LIF signal twice: the first time with an excitation pulse at a low radiance, and the second time with an excitation pulse at a high radiance. The first LIF signal is free from ASE distortion but is noisy due to the low excitation radiance. In contrast, the second LIF signal has high SNR but is distorted due to the ASE effects triggered by the high excitation radiance. Our proposed method combines these two measurements to produce a faithful LIF measurement with high SNR. This paper explains the method and its underlying physics in detail and reports numerical results to demonstrate its application in flow diagnostics. Finally, this paper also examines the practical considerations for implementing the method. These results are expected to be useful for the design and analysis of experiments involving TPLIF and for the expanded use of TPLIF for quantitative flow measurements.

The rest of the paper is organized as follows: Section 2 describes the MC model that is used in this work and is followed by an illustration of the ASE effects in TPLIF measurements in Section 3. Section 4 discusses the correction method to quantify the LIF signals using two measurements. Finally, Section $\underline{5}$ summarizes the paper.

\section{Description of Monte Carlo Model}

Figure 1(a) illustrates the major processes in TPLIF, which involves a four-level system interacting with a laser pulse. An excitation laser pulse excites the target species from the ground level (level 1, with population denoted as $n_{1}$, and the same notation is used hereafter) to the excited level (level 3) via two-photon absorption. Atoms on the excited level can either absorb an additional photon to be ionized (level 4) or fluorescence to state 2 , emitting a LIF photon. Then the LIF photon, as described in Section 1, triggers the ASE process when a population inversion exists. The coordinate system used in this work is also shown in Fig. 1(a), with the positive $x$ axis defined in the propagation direction of the excitation laser pulse. These four levels are also coupled via collisional quenching and stimulated and spontaneous emission (not shown in Fig. 1). Spontaneous emission was not considered due to its relatively slow rate compared to other processes at the laser intensity

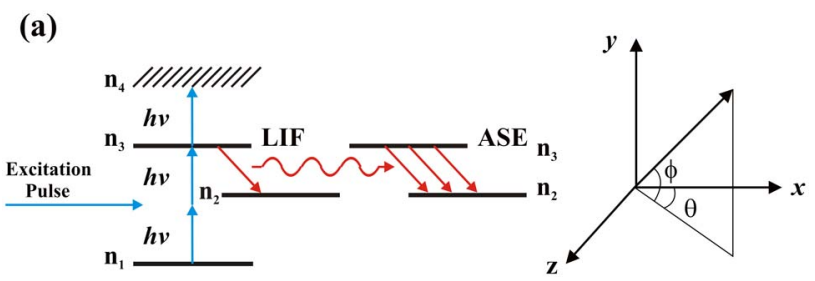

(b)

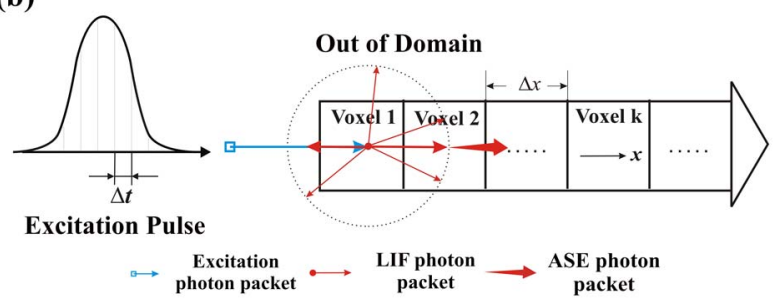

Fig. 1. (Color online) (a) Illustration of the TPLIF process and ASE effects. (b) Schematic of the MC model in 1D; schematic of the MC model in multidimensional. 
under consideration here. All other processes were included in the mode.

The goal of any model development is to consider all of the processes described in this section and to predict the temporal and spatial profiles of all the relevant physical properties, including the population at each level, the LIF photons, and the radiation field. Based on such understanding, various approaches have been proposed to model the TPLIF process. In this work, we used the MC method described in [25] due to its simplicity in implementation and its ability to incorporate non-ideal effects in the model.

Here we provide a brief summary of the MC model with the aid of Fig. 1(b). More detailed description is provided in [25]. The measurement domain is discretized into voxels. Figure 1(b) uses a 1D array of voxels (with dimension $\Delta x$, as shown) to explain the model, and extension of the MC model into multidimension is straightforward. The excitation pulse is discretized both temporally (with a step size of $\Delta t$, as shown, and $\Delta t=\Delta x / c$, where $c$ represents the speed of light) and spatially so that arbitrary excitation profile can be considered in the model. The excitation pulse is modeled as $N$ photon packets, where $N=T / \Delta t$, with $T$ representing the total duration of the excitation pulse.

The model starts by sending the first excitation photon packet into the first voxel. The absorption of this packet in the first voxel by the target species is calculated according to the four-level model shown in Fig. 1(a). The populations at all four levels (i.e., $n_{1}$ through $n_{4}$ ) in the first voxel due to the absorption are then updated. Next, the number of LIF photons emitted in the first voxel during time $\Delta t$ is calculated using the equation $N^{\mathrm{LIF}}=\left.n_{3,1}\right|_{t=\Delta t} \cdot A_{32} \cdot \Delta t$, where $n_{i, k \mid t}$ represents the population of the target species on level $i$ in voxel $k$ at time $t$, and $A_{32}$ is the Einstein $A$ coefficients between states 3 and 2 . These LIF photons are emitted randomly in all directions. Our MC model tracks these photons by 1 ) randomly generating $M$ directions, and 2) dividing these LIF photons into $M$ packets, with each packet propagating in a direction generated in step 1 . At this point, the MC model updates the populations at all levels in all voxels, the number of photons left in the first excitation packet, and the number and direction of all LIF photon packets.

The second packet of excitation photons is then sent into the first voxel cell, and calculations similar to those described in the above paragraph are repeated for these photons in the first voxel. The number of photons absorbed, the LIF photons emitted, and the populations at all levels are determined and updated. All these calculations are performed for the second time step (i.e., for time $t=2 \Delta t$ ). For the remaining photons in the first excitation packet (i.e., those transmitted through voxel 1), the MC model advances their position into voxel 2 and performs the same calculations. For the LIF photon packets generated by the first excitation packet (in voxel 1), the MC model advances their position by $\Delta x$ in the directions generated above and determines whether they exit the computational domain [as shown in Fig. 1(b)]. If a packet exits the computational domain, the MC model stops tracking it. If not, the new location of the LIF packet is determined, and the ASE photons generated by the LIF photon packet in voxel 2 over a gain length of $\Delta x$ are calculated. The ASE photons propagate in the same direction as the LIF photon packet that generates them.

At this point, the MC model updates the population on each level and each cell $\left(n_{i, k}\right)$, the LIF photon packets and their directions, and the ASE photon packets and their directions. Temporally, such updates register the cumulative effects due to the first and second packets of excitation photons. Spatially, such effects are limited within the first two voxels.

In this manner, subsequent packets of excitation photons are sent in one packet at a time. With the incident of each new excitation packet, the MC model 1) advances the spatial positions of the remaining photon packets (excitation, LIF, and ASE) caused by previous excitation packets by $\Delta x, 2$ ) advances the temporal step by $\Delta t$, and 3) repeats the absorption, LIF emission, and ASE emission calculations described above. The MC model terminates when all photon packets (excitation, LIF, and ASE) have exited the computational domain. Extension of the MC model to the multidimensional domain is straightforward and has been detailed [25].

Figure 2 shows a set of sample results generated by the $\mathrm{M} \overline{\mathrm{C}}$ model to simulate the LIF and ASE signals generated by $\mathrm{H}$ atoms in an $\mathrm{H}_{2} / \mathrm{O}_{2} / \mathrm{Ar}$ flame [11]. Our MC model differentiates LIF and ASE signals: LIF signal is due to photons spontaneously emitted from state 3 to 2 , and ASE signal is due to photons emitted by stimulated transitions from state 3 to 2. Specifically, in Fig. 2 the LIF signal represents LIF photons collected at a right angle, and the ASE signal represents ASE photons collected in the forward direction. Parameters used in the MC model

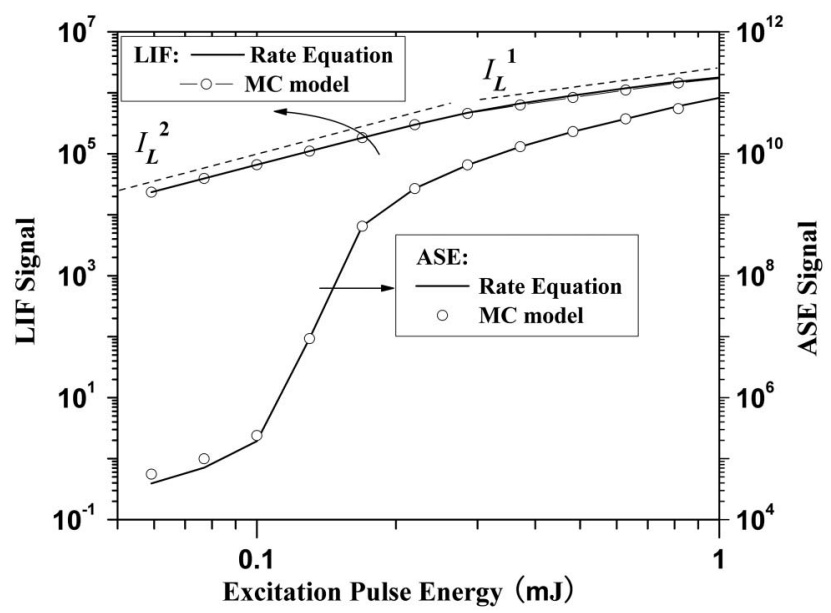

Fig. 2. Comparison of the LIF and ASE signals calculated by the $\mathrm{MC}$ model and the rate equation. Calculations conducted to simulate the LIF and ASE signals from $\mathrm{H}$ atoms in an $\mathrm{H}_{2} / \mathrm{O}_{2} / \mathrm{Ar}$ flame. 
were matched to the experimental conditions as described in [11]. Signals simulated by the MC model agree with the past experimental data both qualitatively and quantitatively. Qualitatively, the MC results agree with the well-known trend of the LIF and ASE signals $[4,11,16,19,26]$. For instance, the LIF signal first scales as $\bar{I}_{L}^{2}$ when the excitation is weak; then, due to the increasing depopulation of level 3 caused by ionization and ASE at strong laser field, scaling gradually becomes as the excitation energy increases. Note that the dashed lines on Fig. 2 are only used to show the $I_{L}^{2}$ and $I_{L}^{1}$ scaling. Quantitatively, the experiments in [11] showed a 20-30x increase in the ASE signal when the excitation energy increased from 0.2 to $0.6 \mathrm{~mJ}$, compared to an $\sim 20 x$ increase predicted by the calculations. Simulations were also conducted using the $1 \mathrm{D}$ rate equation extensively used previously [14-21]. The results, also shown in Fig. $\underline{2}$, are in good agreement with the MC model.

Comparison of the MC model against previous experiments and the rate equation was also performed for the $\mathrm{H}$ atom, under the conditions where experimental data are available $[\underline{4}, 16]$. Good agreement similar to that shown in Fig. 2 was obtained in all cases. The good performance in these cases was attributed to the fact that the measurement domain can be accurately approximated by a 1D domain. For example, in the $\mathrm{H}$ atom experiments [11], the measurement domain had a length of $\sim 3 \mathrm{~cm}$ and a radius of $\sim 120 \mu \mathrm{m}$, resulting in an aspect ratio of $\sim 240$. As the aspect ratio of the measurement domain decreases, the error caused by the 1D assumption in the rate equation increases [25]. In this work, the MC model was chosen for its straightforward extension to multidimensional. Other considerations that motivate the use of the MC model include its simple implementation, the flexibility to incorporate non-ideal conditions (realistic geometries, laser profile, and optical components), and its ability to generate quantities that are difficult or even infeasible to obtain either experimentally or by the rate equation. These advantages will greatly facilitate the inpractice application of the method developed here.

In summary, this section describes the MC model used for this study. The MC model was validated by its good agreement with previously published experimental data and the rate equations. The next section reports results obtained using the MC model to illustrate the effects of ASE in TPLIF measurements.

\section{ASE Effects in TPLIF Measurements}

To illustrate the effects of ASE in TPLIF measurements, the MC model was applied to a simple case where $n_{1}$ assumes a uniform (i.e., top-hat) distribution. The measurement domain was taken to be cylindrical with a length of $3 \mathrm{~cm}$ and a diameter of $250 \mu \mathrm{m}$, simulating typical conditions for 1D measurements in a laboratory flame.

Figure 3 shows the LIF and ASE signals calculated by the $\overline{\mathrm{MC}}$ model for the $\mathrm{H}$ atom at an excitation

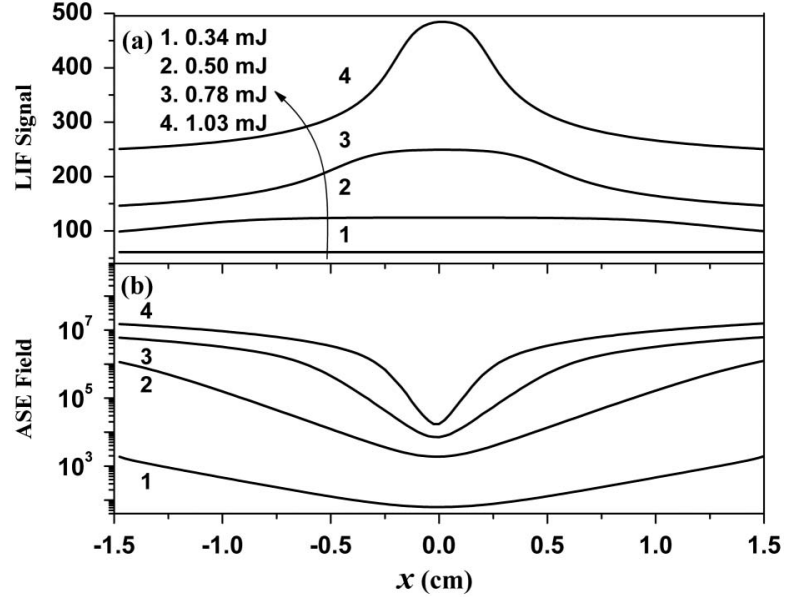

Fig. 3. LIF and ASE signals calculated by the MC model at various excitation pulse energies. The ASE field was represented by the number of ASE photons in each voxel at a time of $4 \mathrm{~ns}$.

wavelength of $205 \mathrm{~nm}$ and LIF/ASE photons at $656 \mathrm{~nm}$. The ground state number density of the $\mathrm{H}$ atom $\left(n_{1}\right)$ was set to be $8.5 \times 10^{14} \mathrm{~cm}^{-3}$. This value was obtained using an equilibrium calculation for an $\mathrm{H}_{2} / \mathrm{O}_{2} / \mathrm{Ar}$ flame. The excitation laser pulse was assumed to have a Gaussian temporal profile with an FWHM of $3.5 \mathrm{~ns}$ [19], the linewidth was assumed to be $6.88 \mathrm{~cm}^{-1}$ according to [27], and the diameter of the

laser beam was assumed to be $250 \mu \mathrm{m}$. The quenching rates from levels 2 and 3 were determined to be $8.1 \times 10^{8} \mathrm{~s}^{-1}$ according to [28]. Other spectroscopic parameters are summarized in Table 1 . In the MC model, the domain of interest ( $3 \mathrm{~cm}$ in length, $250 \mu \mathrm{m}$ in diameter) was discretized into 120 cylindrical grids in the $x$ direction. The grid's size was thus $250 \mu \mathrm{m}$ in length and $250 \mu \mathrm{m}$ in diameter. The time step $\Delta t$ is set to be 0.83 ps.

The results shown in Fig. 3(a) are intended to illustrate the signal level of a typical TPLIF measurement in practice. The LIF signal is measured in number of photons per pixel. In obtaining the LIF signal, the following parameters were assumed: an imaging system with $0.1 \%$ collection efficiency and $50 \%$ overall quantum efficiency, an integration time of $10 \mathrm{~ns}$, a magnification of unity, and a pixel size of $10 \times 10 \mu \mathrm{m}^{2}$. Figure $3(\mathrm{a})$ shows the LIF signal at four excitation energies that were chosen to elucidate the onset and saturation of the ASE effects. At low excitation energy (case 1), the ASE field [shown in Fig. 3(b) in units of ASE photons per voxel] was too low to generate appreciable distortion, and a flat

Table 1. Spectroscopic Properties of the H Atom Used in This Work

\begin{tabular}{lclc}
\hline Parameters & \multicolumn{1}{c}{ Values } & Units & References \\
\hline $\begin{array}{l}\text { Two-photon absorption } \\
\text { cross section }\end{array}$ & $1.17 \times 10^{-28}$ & $\mathrm{~cm}^{4} / \mathrm{W}$ & {$[27]$} \\
$\begin{array}{l}\text { Ionization cross } \\
\text { section }\end{array}$ & $9.0 \times 10^{-20}$ & $\mathrm{~cm}^{2}$ & {$[30]$} \\
$A_{32}$ & $2.89 \times 10^{7}$ & $\mathrm{~s}^{-1}$ & {$[31]$} \\
$A_{21}$ & $4.7 \times 10^{8}$ & $\mathrm{~s}^{-1}$ & {$[\underline{31}]$} \\
\hline
\end{tabular}


LIF signal was observed, faithfully representing the true uniform distribution to be measured. As the excitation energy increases (cases 2,3 , and 4 ), the ASE fields grow rapidly and induce transition between $n_{2}$ and $n_{3}$ to compete with the quenching process. As a result, evident distortions in the LIF signal were observed. The LIF and ASE signals for these cases are shown in Fig. 4 to illustrate the transition from the onset to the saturation of the ASE effects. The ASE signal in Fig. 4 is measured in photons per pulse, and the LIF signal is measured in photons per pixel.

The results shown in Fig. 3 also illustrate the dilemma in TPLIF applications. In practice, researchers typically design experiments to avoid the onset of significant ASE by using low excitation energy or by limiting the number density of target species to be measured. The LIF signal obtained in this case is free from distortion, at the cost of reduced signal level and hence low SNR. As shown in Fig. 3, the LIF signal increased by a factor of $\sim 8 \times$ when the excitation laser energy increased from 0.34 to $1.03 \mathrm{~mJ}$. The complication in this case is that the LIF signal is distorted and no longer represents the true distribution to be measured. The correction of such distortion is challenging because the distortion is nonlinear (as shown here) and nonlocal; i.e., the distortion at one location depends on the conditions (such as $n_{1}$ and temperature) at other locations. In this aspect, the correction of the ASE distortion is more difficult than the correction of quenching rate and ionization. The correction of such distortion is the topic of the next section.

\section{Correction of ASE Distortion in TPLIF Measurements}

\section{A. Introduction of Correction Method}

Figure 5 illustrates the dilemma in TPLIF measurements discussed in Section 3. The LIF signals at relatively low and high excitation pulse energies

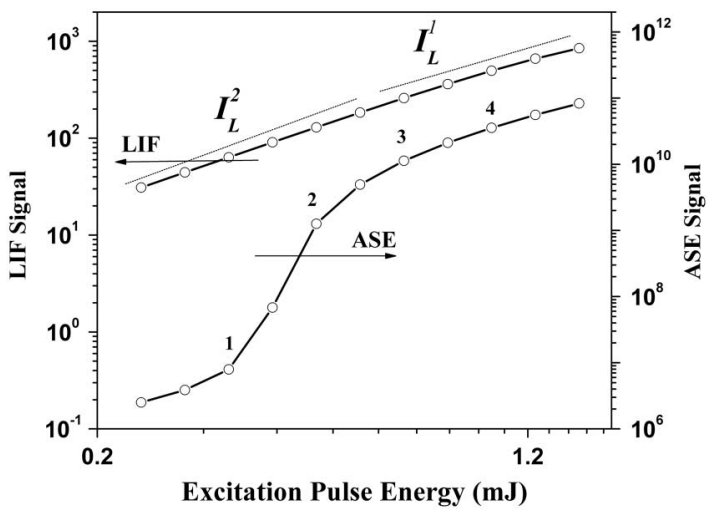

Fig. 4. LIF and ASE signals simulated for $\mathrm{H}$ atoms. The LIF signal corresponds to the number of LIF photons received on the voxel corresponding to $x=0$. The ASE signal corresponds to the ASE photons received in the forward direction. An integration time of $10 \mathrm{~ns}$ was used for the calculation of both the LIF and ASE signals. are denoted as $S_{L}$ and $S_{H}$, as shown in Fig. 5(a). Artificial noises were generated and added to the LIF signals to simulate practical measurements according to the following equations:

$$
S_{L N}=P\left(S_{L}\right)+\epsilon \quad \text { and } \quad S_{H N}=P\left(S_{H}\right)+\epsilon,
$$

where $S_{L N}$ and $S_{H N}$ represent the measured LIF signals with noise at low and high excitation energies, respectively; $P(S)$ represents a Poisson noise with an expectation of $S$ and standard deviation of $\sqrt{S}$ to simulate the shot noise; and $\varepsilon$ represents a Gaussian random noise with an expectation of zero and standard deviation of 10 counts to simulate the readout noise and dark noise typical of current CCD devices. Figure 5(a) shows the simulated signals with and without noise, and Figs. 5(b) and 5(c) show the relative noise for the LIF signals measured at low and high excitation energies, defined as $S_{L N} / S_{L}$ and $S_{H N} / S_{H}$, respectively. As discussed above, the signal obtained at high excitation energy enjoys a low noise (about $\pm 5 \%$ ) compared to the signal obtained at low excitation energy (about $\pm 30 \%$ ). However, the signal at high excitation energy is distorted.

Now we describe a method to correct the distorted signal and obtain a faithful measurement with high SNR. The method uses the LIF signals measured both with low and high excitation energies, as shown in Fig. 5(a). Consequently, this method requires measuring the TPLIF signal twice: once with a low excitation energy, and the second time with a high excitation energy. Admittedly, this requirement will result in additional implementation complication relative to the typical TPLIF setup; however, the complication should be manageable. For the laser, a straightforward way of obtaining two measurements is to use two lasers to generate the two excitation pulses. Or, alternatively, the output from one laser can be split into two beams, one with a low energy and one with a high energy. An optical delay is then introduced between these two beams to take the

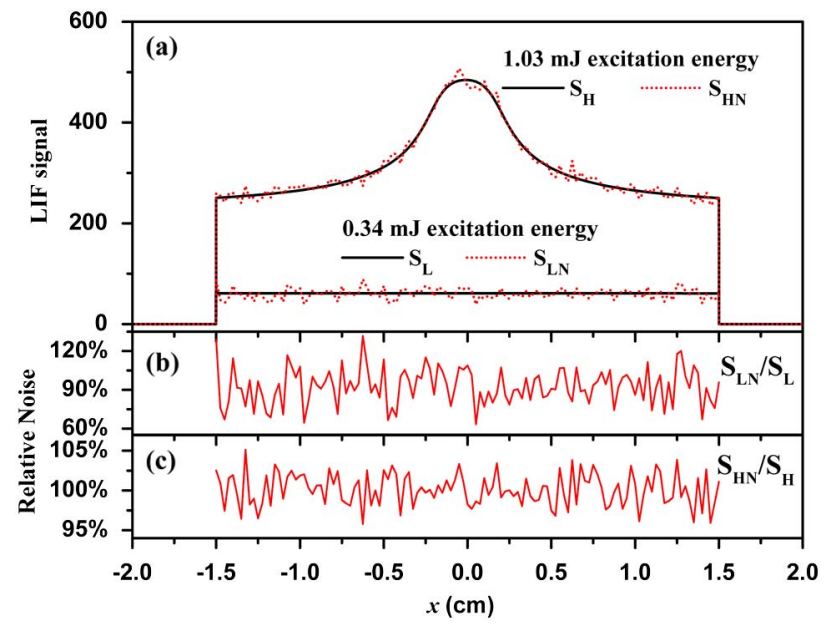

Fig. 5. (Color online) LIF signals at relatively low and high excitation energies, with artificial noise added to simulate practical measurements. 
measurements sequentially. Either two cameras or one camera with a double frame feature can be used to capture the required measurements.

Our method to correct the distortion caused by ASE is built on an argument that is derived from the physics of ASE and has been confirmed by numerical simulations. The argument is that the ratio between the LIF signals obtained at low and high excitation energies has a relatively stable and smooth shape. As shown in Fig. 6(a), the ratio $\left(R_{\text {True }}\right.$, defined as $S_{L} / S_{H}$ ) has an inverted bell shape. This shape (and its smoothness) is insensitive to the distribution of the target species to be measured (i.e., $n_{1}$ ) and to the energy of the excitation pulses. This argument has been confirmed by extensive numerical simulations, and results for other example simulations will be shown later in this section. Here we explain the argument based on the physics of ASE. The shape of the ratio will be explained first and then the smoothness of the ratio.

\section{B. Shape of Ratio}

The inverted bell shape is caused by the fact that the ASE effects are stronger at the two ends of the measurement region than in the middle because the two ends offer more effective gain length [29]. Consequently, the depopulation will be more significant at the two ends than in the middle, in turn resulting in weaker LIF signal at the two ends than in the middle. Therefore, the distorted LIF signal, when normalized by an undistorted signal, exhibits the inverted bell shape shown in Fig. 6(a). Here the flatness of the undistorted signal (due to the top-hat $n_{1}$ distribution) helps to elucidate this intuitive argument. The argument also holds for other distributions of $n_{1}$.

Further insights from this argument can be explained through analysis of the ASE radiation equations. For the sake of brevity, here we analyze the equations under steady state. Similar analysis can be performed for general cases. Under steady state,

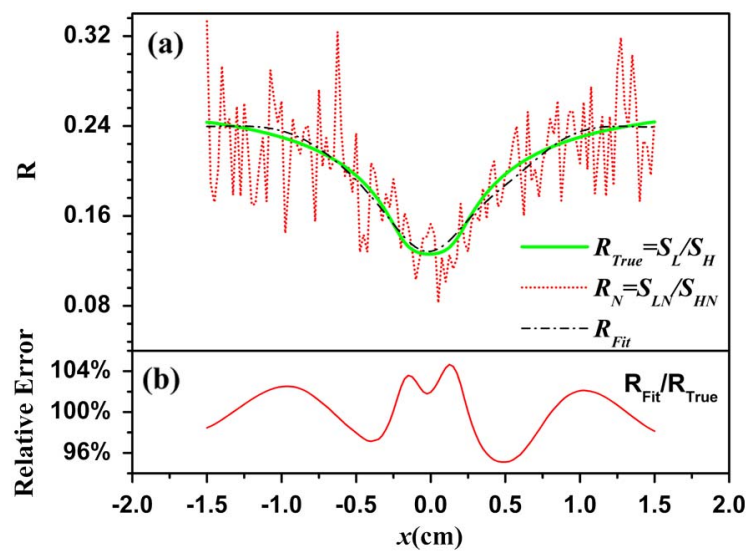

Fig. 6. (Color online) (a) Ratio between the LIF signals obtained at low and high excitation energies. (b) The relative error in the fitted ratio. the gradient of the total ASE field $\left(I_{\mathrm{ASE}}\right)$ can be written as $[\underline{16}, \underline{19}]$

$$
\begin{aligned}
\frac{\partial}{\partial x} I_{\mathrm{ASE}}(x)= & \frac{B_{32} \Gamma}{c \Delta v_{\mathrm{ASE}}}\left(I_{\mathrm{ASE}}^{f}(x)-I_{\mathrm{ASE}}^{b}(x)\right) \Delta n \\
& +A_{32}\left(\Delta \Omega_{f}(x)-\Delta \Omega_{b}(x)\right) n_{3}(x) h v_{\mathrm{ASE}}, \\
& \Delta n=\left(n_{3}(x)-\frac{g_{3}}{g_{2}} n_{2}(x)\right),
\end{aligned}
$$

where represents the population inversion; $g_{i}(i=1$, $2,3,4)$ represents the degeneracy of each level; $B_{32}$ represents the Einstein $B$ coefficient from state 3 to state $2 ; \Gamma$ represents the overlap integral defined as $\int_{-\infty}^{+\infty} g(v) f(v) \mathrm{d} v$, with $g(v)$ and $f(v)$ representing the line shape function of the absorption transition and the ASE radiation, respectively; $\Delta v_{\mathrm{ASE}}$ represents the linewidth of the ASE radiation; $I_{\mathrm{ASE}}^{f}$ and $I_{\mathrm{ASE}}^{b}$ represent the irradiance of the ASE photons in the forward (i.e., positive $x$ ) and backward (i.e., negative $x$ ) directions, respectively; $A_{32}$ represents the Einstein A coefficient for transition from state 3 to state $2 ; \Delta \Omega_{f}$ and $\Delta \Omega_{b}$ represent the solid angle formed by the incident and exit surface of the domain of interests relative to the point at which the ASE photons are emitted; $h$ represents the Planck constant; and $\nu_{\mathrm{ASE}}$ represents the frequency of the ASE photons.

As can be seen, $I_{\mathrm{ASE}}^{f}(x), I_{\mathrm{ASE}}^{b}(x)$, and $I_{\mathrm{ASE}}(x)$ are coupled by Eq. (2). In the first term on the right-hand side of Eq. (2), $I_{\mathrm{ASE}}^{\bar{f}}(x)$ increases monotonically with $x$ while $I_{\mathrm{ASE}}^{b}(x)$ decreases. Therefore, the first term starts from a negative value at the incident end and grows to a positive value at the exit end. The same trend also applies to the second term, i.e. $\left[\Delta \Omega_{f}(x)-\right.$ $\Delta \Omega_{b}(x)$ ], from simple geometrical consideration. Hence, $I_{\mathrm{ASE}}(x)$ starts with a negative gradient from the incident end and transitions to a positive gradient at the exit end, leading to the inverted bell shape of the ratio. Also note that $\Delta \Omega_{f}(x)-\Delta \Omega_{b}(x)$ at the center of the measurement domain, which suggests that the minimum of $I_{\mathrm{ASE}}(x)$, and therefore the tip of the bell, tends to be located at the middle of the measurement domain. The location will be exactly at the middle point $I_{\mathrm{ASE}}^{f}(x)$ if also equals $I_{\mathrm{ASE}}^{b}(x)$ there (e.g., in the case of a symmetric $n_{1}$ distribution).

\section{Smoothness of Ratio}

Having explained the shape of the ratio curve, we now examine its smoothness. This curve is smooth even if the $n_{1}$ distribution fluctuates significantly spatially, because the ASE effects are accumulative along the path, and as a result, the fluctuations (or discontinuities) in $n_{1}$ will be smoothed out. This reasoning can be analyzed mathematically using the ASE radiation equations. Here again, we analyze it under steady state for the sake of brevity. Similar 
analysis can be undertaken for general cases. Under steady state, Eq. (2) is the governing equation for the ASE field. If only the field caused by ASE photons in the forward direction is considered, then Eq. (2 $)$ is modified to

$$
\begin{aligned}
\frac{\partial}{\partial x} I_{\mathrm{ASE}}^{f}(x)= & \frac{B_{32} \Gamma}{c \Delta \nu_{\mathrm{ASE}}} \cdot I_{\mathrm{ASE}}^{f} \cdot \Delta n+A_{32} \cdot \Delta \Omega_{f}(x) \\
& \cdot n_{3}(x) \cdot h \nu_{\mathrm{ASE}} .
\end{aligned}
$$

Based on Eq. (4), we will show that the ratio caused by $I_{\mathrm{ASE}}^{f}(x)$ is smooth, and a similar analysis can be made for $I_{\mathrm{ASE}}^{b}$.

Two assumptions can be made to simplify Eq. (무) and obtain an analytical solution: (1) $\Delta n$ is assumed

$$
W_{32}^{f}=\frac{B_{32} I_{\mathrm{ASE}, f}}{c \Delta \nu_{\mathrm{ASE}}} \Gamma \quad \text { and } \quad W_{32}^{b}=\frac{B_{32} I_{\mathrm{ASE}, b}}{c \Delta \nu_{\mathrm{ASE}}} \Gamma,
$$

where $W_{13}$ and $W_{34}$ represent the transition rate coefficients of two-photon absorption and ionization, respectively; $W_{32}^{f}$ and $W_{32}^{b}$ represent the transition rate coefficients between levels 2 and 3 caused by the ASE photons in the forward and backward directions, respectively; and $Q_{3 a}$ represents the collisional quenching rates from state 3 to all other states. Since we are only analyzing the effects caused by the ASE photons in the forward direction, $W_{32}^{b}$ does not concern us in this analysis. Substituting Eq. (5) into Eq. (7) and solving Eq. (6) yields

$$
n_{3}(x)=\frac{W_{13} n_{1}(x)}{\frac{A_{32} \Delta \Omega_{f}}{B_{32} \Gamma / c \Delta \nu_{\mathrm{ASE}}}\left[\exp \left(\frac{B_{32} \Gamma}{c \Delta \nu_{\mathrm{ASE}}} \int_{0}^{x} n_{3}(x) \mathrm{d} x\right)-1\right]+\left(W_{34}+A_{32}+Q_{3 a}\right)+W_{13} \frac{g_{1}}{g_{3}}} .
$$

to be equal to $n_{3}$ because $n_{2}$ is typically insignificant compared to $n_{3}$, and (2) $\Delta \Omega_{f}(x)$ is assumed to be independent of $x$. The essence of this analysis is to show that the ASE effects and the ratio are smooth when $n_{1}$ is not. These assumptions are needed only to simplify the algebra, and they do not change the essence of this analysis (unless $\Delta \Omega_{f}(x)$ itself is a nonsmooth or discontinuous function). Under these assumptions, solving Eq. (4) with the boundary condition $I_{\mathrm{ASE}}^{f}(0)=0$ yields

$$
\begin{aligned}
I_{\mathrm{ASE}}^{f}(x)= & \left.\frac{A_{32} \Delta \Omega_{f} h \nu_{\mathrm{ASE}}}{B_{32} \Gamma / c \Delta \nu_{\mathrm{ASE}}} \exp \left(\frac{B_{32} \Gamma}{c \Delta \nu_{\mathrm{ASE}}} \int_{0}^{x} n_{3}(x) \mathrm{d} x\right)\right) \\
& -\frac{A_{32} \Delta \Omega_{f} h \nu_{\mathrm{ASE}}}{B_{32} \Gamma / c \Delta \nu_{\mathrm{ASE}}} .
\end{aligned}
$$

Equation (5) illustrates that the ASE effects do not directly depend on $n_{3}(x)$, which can be nonsmooth or discontinuous. Instead, the ASE effects depend on $\int_{0}^{x} n_{3}(x) \mathrm{d} x$. Therefore, even when $n_{3}(x)$ is nonsmooth or discontinuous [rooted from $n_{1}(x)$ ], the integration smoothes out the distribution.

The rate equation for the population on state 3 is

$$
\begin{aligned}
0= & W_{13}\left(n_{1}-\frac{g_{1}}{g_{2}} n_{3}\right)-\left(W_{32}^{f} W_{32}^{b}\right)\left(n_{3}-\frac{g_{3}}{g_{2}} n_{2}\right) \\
& -\left(W_{34}+A_{32}+Q_{3 a}\right) n_{3},
\end{aligned}
$$

As mentioned earlier, $n_{3}(x)$ can be nonsmooth and discontinuous, as its shape correlates to that of $n_{1}(x)$. The ratio between LIF signals obtained at low and high excitation energies (i.e., $R_{\text {True }}$ ) is then

$$
R_{\text {True }}=\frac{n_{3, L}(x)}{n_{3, H}(x)}
$$

where $n_{3 . L}(x)$ and $n_{3 . H}(x)$ represent the population of state 3 caused by the excitation pulse with low and high energies, respectively. When Eq. (8) is substituted into Eq. (9), the $n_{1}(x)$ on the numerator of Eq. (8) is canceled, and $R_{\text {True }}(x)$ depends on $\int_{0}^{x} n_{3, L}(x) \mathrm{d} x$ and $\int_{0}^{x} n_{3 . H}(x) \mathrm{d} x$, which are first-order continuous due to the integration. As a result, $R_{\text {True }}(x)$ is smooth and continuous even when $n_{1}(x)$ is not.

The above analysis also illustrates that $\int_{0}^{x} n_{3}(x) \mathrm{d} x$ is a fundamental and useful parameter in the analysis of ASE effects. However, in practice, $n_{3}(x)$ is usually not the target quantity to be measured, nor is it directly available. The LIF signals (i.e., $S_{L N}$ and $\left.S_{H N}\right)$ are directly available. For practical purposes, we denote $\int_{0}^{x} n_{3}(x) \mathrm{d} x$ by $X$ and argue that it can be approximated by $\int_{0}^{x} S_{L N}(x) \cdot \mathrm{d} x$ due to the following two considerations: first, under low excitation energy, $n_{3}(x)$ will be proportional to $n_{1}(x)$. As elucidated in Eq. (8), under weak excitation, $n_{3}(x)$ approaches zero, causing $\int_{0}^{x} n_{3}(x) \mathrm{d} x$ to approach zero too. Therefore, the first term in the denominator of 
Eq. (8) vanishes, leaving $n_{3}(x)$ to be proportional to $n_{1}(x)$. As a result, is proportional to. Second, under weak excitation, the LIF signal is free from distortion and is therefore proportional to $n_{1}(x)$. The use of in the analysis of TPLIF measurements will be illustrated in Subsection 4.D. The accuracy of approximating $\int_{0}^{x} n_{3}(x) \mathrm{d} x$ with $X$ obviously depends on the noise level in $S_{L N}$, and a thorough investigation merits a separate publication.

\section{Performance of Correction Method}

Based on the analysis explained in Subsection 4.C, the experimentally measured ratio can be fitted into a smooth curve with an inverted bell shape to retrieve the true ratio. As shown in Fig. 6(a), as a result of the measurement noise, especially the noise in the signal obtained with low excitation energy, the experimentally measured ratio (defined as $R_{N}=$ $S_{L N} / S_{H N}$ ) may not be smooth and may not appear to be an inverted bell curve. Smoothing and fitting this noisy ratio can retrieve the true ratio based on our argument above. Here we used a simple thirdorder spline method to obtain the fitted ratio, $R_{\text {Fit }}$. A more elaborate fitting method can improve the fitting quality. Fig. $6(\mathrm{~b})$ shows the relative error in the fitting (quantified by $R_{\mathrm{Fit}} / R_{\text {True }}$ ), which shows that the fitting retrieved the ratio within $\pm 4 \%$.

After the ratio is retrieved, it is used to correct the distorted LIF signal (by multiplying it). The results of such correction are shown in Fig. 7. Figure 7(a) compares the corrected LIF signal (labeled $S_{C}$ ) to $S_{L N}$ and to the ideal signal (labeled $S_{\text {True }}$ ), the signal with neither noise nor distortion. Figure 7(b) illustrates that the noise in the corrected signal is within $\pm 10 \%$, which is significantly lower than the noise in $S_{L N}$. The noise in $S_{C}$ consists of two parts: the first part is the noise in $S_{H N}$, which can be reduced by increasing the excitation energy. The second part is due to the discrepancy between the fitted ratio and the true ratio, as shown in Fig. 6. A more elaborate fitting method can reduce this discrepancy.

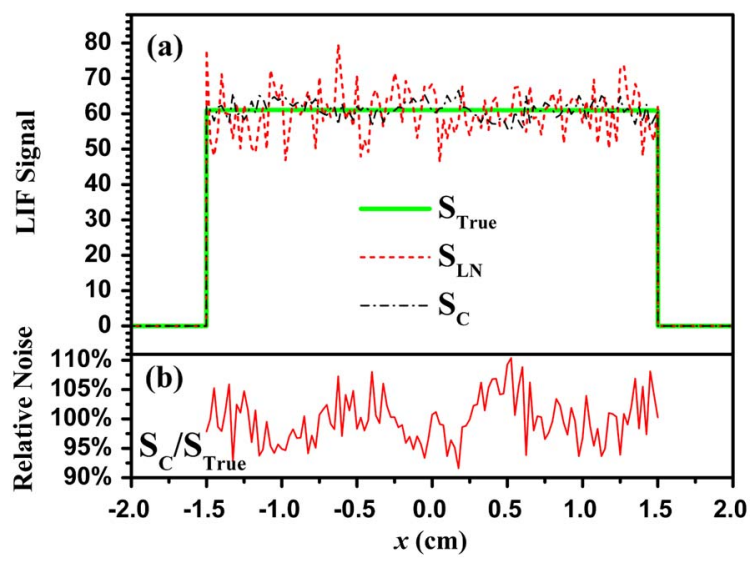

Fig. 7. (Color online) (a) Comparison of the corrected LIF signal to $S_{L N}$ and $S_{\text {True }}$. (b) Illustration that the noise in the corrected signal is significantly lower than the noise in $S_{L N}$.

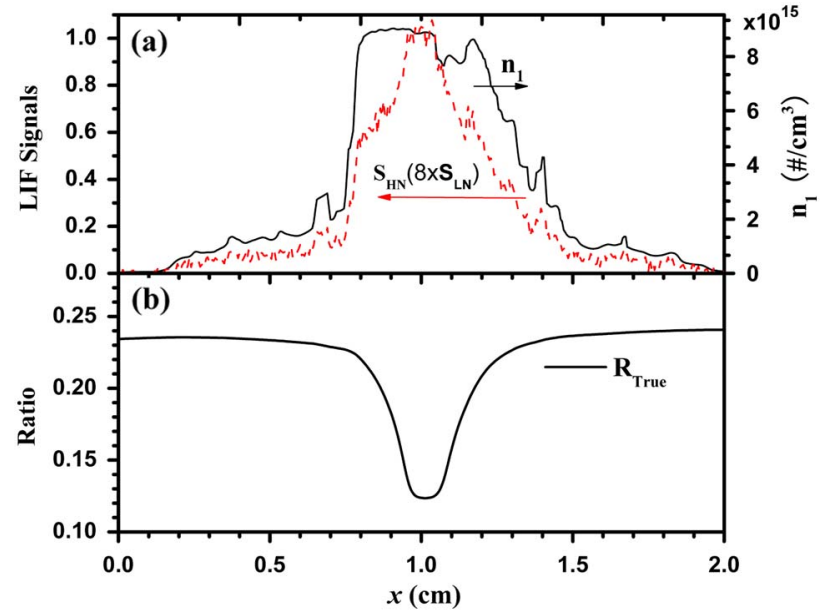

Fig. 8. (Color online) (a) Illustration of the phantom $n_{1}$ distribution used and the distortion caused by ASE. (b) Illustration that the shape of the ratio is insensitive to the $n_{1}$ distribution and the excitation energies.

A more accurate measurement at low excitation energy will also help.

Figures $8-10$ show another set of sample results obtained under a different set of conditions. Figure 8(a) shows the $n_{1}$ distribution of the $\mathrm{H}$ atom. This phantom distribution was taken from a measurement of a conserved scalar in a turbulent jet to simulate the fluctuations in both large scale and small scale. The measurement domain was taken to be cylindrical with a length of $2 \mathrm{~cm}$ and a diameter of $300 \mu \mathrm{m}$. The excitation pulse was assumed to be Gaussian with FWHM of $3.5 \mathrm{~ns}$. The low and high excitation energies used were 0.059 and 0.169 mJ. The peak $H$ number density was designed to be higher by $\sim 10 \times$ than in the previous case; therefore, lower excitation energies were used. These parameters were chosen to illustrate that the argument holds under different conditions. Figure $8\left(\right.$ a) shows $S_{H N}$ scaled by a factor of $1 / 8$, obtained according to Eq. (1) to illustrate the distortion caused by ASE. The $S_{H N}$ is $8 \times$ stronger

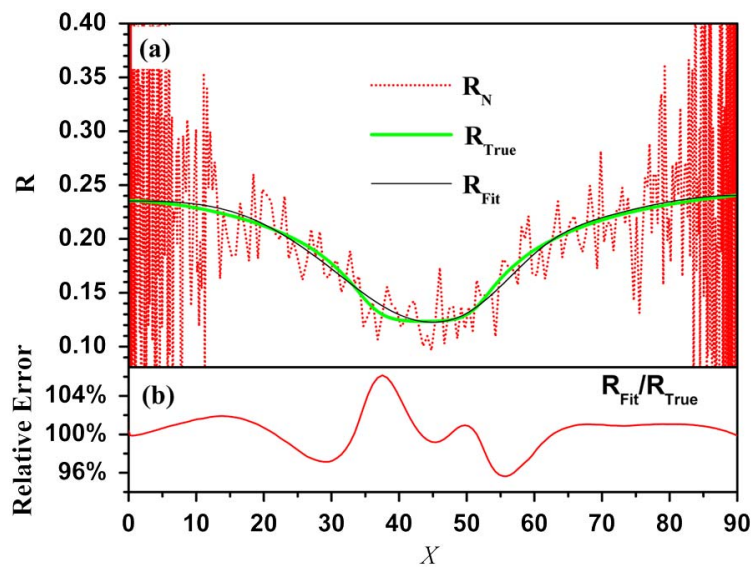

Fig. 9. (Color online) (a) Ratio between the LIF signals obtained at low and high excitation energies. (b) The relative error in the fitted ratio. 


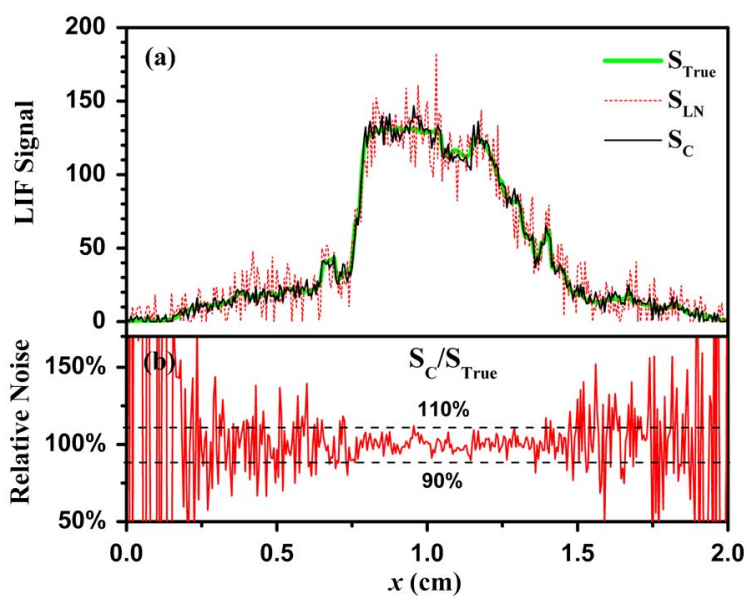

Fig. 10. (Color online) (a) Comparison of the corrected LIF signal to $S_{L N}$ and $S_{\text {True }}$ (b) Illustration that the noise in the corrected signal is significantly lower than the noise in $S_{L N}$.

than $S_{L N}$ in this case, which will cover the $n_{1}$ curve if shown. Figure $8(\mathrm{~b})$ shows the ratio of the LIF signal from low and high excitation energies. As argued above, the ratio is a smooth curve with an inverted bell shape. Figure 9(a) shows the ratios between LIF signals. Note that Fig. 9 used the new parameter $X=\int_{0}^{x} S_{L N}(x) \cdot \mathrm{d} x$, whose units will be the units of the LIF signal (photons per pixel) multiplied by the length $(\mathrm{cm})$. For the analysis of the top-hat distribution, $X$ is equivalent to $x$. Since the physical meanings for $R_{N}, R_{\text {True }}$, and $R_{\text {Fit }}$ are unchanged, we use the same notation for ratios as functions of $X$. Similar to the results shown in Fig. 6 , a third-order spline fit of $R_{N}$ was used to approximate $R_{\text {True }}$. The relative error of this fit was shown in Fig. 6 (b) to be within $\pm 4 \%$. Finally, Fig. 10(a) shows the comparison of the corrected LIF signal to $S_{L N}$ and $S_{\text {True }}$, and Fig. 10(b) illustrates the relative error in the corrected signal. The relative error is within $\pm 10 \%$ in the region $(0.7<x<1.3 \mathrm{~cm})$, where the $n_{1}$ is relatively high, in contrast to $\pm 30 \%$ in $S_{L N}$ in the same region. In regions where $n_{1}$ is low, the relative error is large due to the large noise in both $S_{L N}$ and $S_{H N}$ in these regions. As previously mentioned, increasing the excitation laser pulse can help to reduce errors in these regions.

Thus far, we have described the correction method, its physical background, and its demonstration on two distributions. For these two specific distributions, the correction method has been shown to reduce both noise and distortion. To systematically analyze its applicability and performance, we need to quantitatively define noise and distortion. This work defines noise as

$$
\text { Note }=\frac{1}{L} \int_{L} \frac{\left|S_{L N}-S_{L}\right|}{\left|S_{L}\right|} \mathrm{d} x \text { or } \frac{1}{L} \int_{L} \frac{\left|S_{H N}-S_{H}\right|}{\left|S_{H}\right|} \mathrm{d} x
$$

where $L$ represent the measurement domain. Equation (10) essentially defines a noise averaged in the measurement domain. Distortion is defined as
Distortion $=\frac{1}{L} \int_{L}\left|\frac{S_{L}}{A n_{1}}-1\right| \mathrm{d} x$ or $\frac{1}{L} \int_{L}\left|\frac{S_{H}}{B n_{1}}-1\right| \mathrm{d} x$

This definition quantifies the average deviation of the noise-free measurements $\left(S_{L}\right.$ or $\left.S_{H}\right)$ relative to $n_{1}$. The constants in the equation, $A$ and $B$, are normalization factors determined according to

$$
\int_{L} S_{L}^{2} \mathrm{~d} x=\int_{L}\left(A \cdot n_{1}\right)^{2} \mathrm{~d} x \text { and } \int_{L} S_{H}^{2} \mathrm{~d} x=\int_{L}\left(B \cdot n_{1}\right)^{2} \mathrm{~d} x
$$

These definitions need to be modified for the corrected signal $\left(S_{C}\right)$ because $S_{C}$ contains both the true signal and the noise. Here, we use $S_{H} \cdot R_{\text {Fit }}$ to represent the true signal in $S_{C}$, and noise and distortion are defined correspondingly as

$$
\begin{gathered}
\text { Noise }=\frac{1}{L} \int_{L} \frac{\left|S_{C}-S_{H} \cdot R_{\mathrm{Fit}}\right|}{S_{H} \cdot R_{\mathrm{Fit}}} \mathrm{d} x, \\
\text { Distortion }=\frac{1}{L} \int_{L}\left|\frac{S_{H} \cdot R_{\mathrm{Fit}}}{C \cdot n_{1}}-1\right| \mathrm{d} x .
\end{gathered}
$$

Constant $C$ in the equation is a normalization factor determined according to

$$
\int_{L}\left(S_{H} \cdot R_{\mathrm{Fit}}\right)^{2} \mathrm{~d} x-\int_{L}\left(C \cdot n_{1}\right)^{2} \mathrm{~d} x
$$

With these definitions, simulations were made for many cases under various conditions, including $n_{1}$ distribution, excitation pulse energy, and geometry. The $n_{1}$ distributions used were specifically chosen to represent a wide range of flows. For each case, (1) the LIF signals at low and high excitation energies were simulated according to Eq. (1); (2) the correction method was applied to each case using the simulated

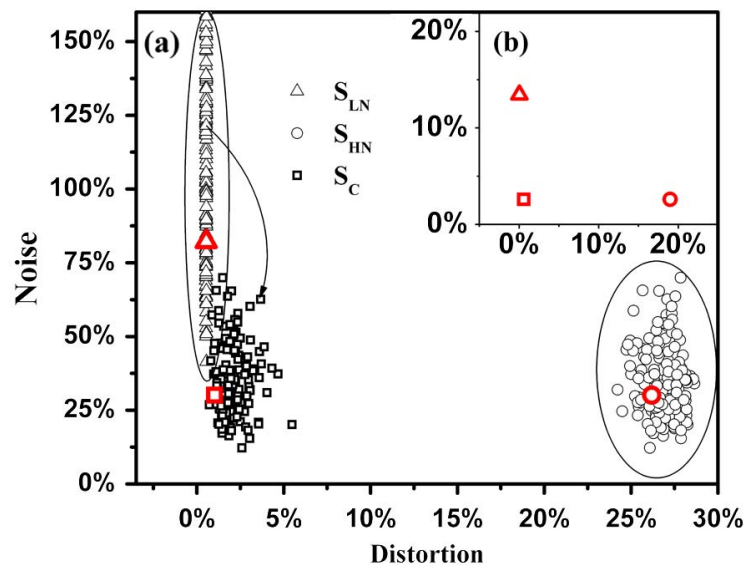

Fig. 11. (Color online) Performance of the correction method simulated for various distributions. (a) The large red symbols correspond to the noise and distortion for the $n_{1}$ distribution shown in Fig. 10. (b) The large red symbols correspond to the noise and distortion of the top-hat distribution shown in Fig. $\underline{5}$. 
signals; and (3) the noise and distortion of the simulated signals $\left(S_{L N}\right.$ and $\left.S_{H N}\right)$ and the corrected signal $\left(S_{C}\right)$ were calculated according to Eqs. (10)-(15). Figure 11 shows the results obtained for $1 \overline{50}$ cases. The large red symbols in Fig. 11(a) correspond to the noise and distortion for the $n_{1}$ distribution shown in Fig. 10; the symbols in Fig. 11(b) correspond to those of the top-hat distribution shown in Fig. 5. As shown here, the correction method reduced both the noise and distortion for all these cases. Note that even though the noise in $S_{C}$ was reduced in all cases compared to the noise in $S_{L N}$, the noise in $S_{C}$ appears to be higher than the noise in $S_{L N}$ for some cases because Fig. 11 does not show the corresponding relationships among the cases. For example, the arrow in Fig. 11(a) illustrates a corrected signal and the $S_{L N}$ it corresponds to. Also note that for some cases, the $n_{1}$ distributions used have some regions with low values, like the two sides of the distribution shown in Fig. 10. The noise in these regions was exceedingly high, causing the high average noise seen in Fig. 11. In practice, measurements made in these regions are usually discarded due to the unacceptably low SNR.

In summary, this section describes the correction method and its physical background. Extensive numerical simulations were conducted to evaluate the performance of the correction method, and the method was demonstrated to be able to reduce noise and distortion in a wide range of conditions.

\section{Summary}

This work examined the effects of ASE in TPLIF measurements using an MC model. The ASE effects represent a major challenge to the application of TPLIF as a flow diagnostic that is more difficult to correct due to its nonlinear and nonlocal nature than quenching and ionization. The ASE effects cause distortion to the target LIF signal, the distortion depends nonlinearly on a range of parameters (e.g., the number density of the target species, laser excitation energy, and the temporal and spatial profiles of the excitation pulse), and the distortion at one location depends on conditions at other locations.

A correction method was developed and demonstrated to correct for the distortion caused by ASE effects. The method was based on a physical understanding of the ASE effect, i.e., the ratio between the LIF signals obtained at low and high excitation energies should have a smooth shape that is insensitive to experimental parameters. Based on this physical understanding, the correction method uses two LIF measurements, one with low SNR and negligible ASE distortion and another with high SNR but significant distortion, to generate a faithful measurement with high SNR. Extensive simulations were performed to evaluate the performance of this method, demonstrating its ability to reduce noise and distortion in TPLIF measurements across a wide range of conditions. We expect this method to be a valuable tool for the application of TPLIF techniques in flow diagnostics. Our ongoing work includes the experimental demonstration of the method and the extension of the scheme to 2D imaging applications.

Funding for this project was provided by the National Science Foundation (Award CBET 0844939).

\section{References}

1. A. C. Eckbreth, Laser Diagnostics for Combustion Temperature and Species (Gordon and Breach, 1996).

2. K. C. Smyth and D. R. Crosley, "Detection of minor species with laser techniques," in Applied Combustion Diagnostics, K. Kohse-Hoinghaus and J. B. Jeffries, eds. (Taylor \& Francis, 2002), pp. 9-68.

3. N. Georgiev and M. Alden, "Two-dimensional imaging of flame species using two-photon laser-induced fluorescence," Appl. Spectrosc. 51, 1229-1237 (1997).

4. J. H. Frank, X. L. Chen, B. D. Patterson, and T. B. Settersten, "Comparison of nanosecond and picosecond excitation for two-photon laser-induced fluorescence imaging of atomic oxygen in flames," Appl. Opt. 43, 2588-2597 (2004).

5. N. J. Bednar, J. W. Walewski, and S. T. Sanders, "Assessment of multiphoton absorption in inert gases for the measurement of gas temperatures," Appl. Spectrosc. 60, 246-253 (2006).

6. A. G. Hsu, V. Narayanaswamy, N. T. Clemens, and J. H. Frank, "Mixture fraction imaging in turbulent non-premixed flames with two-photon LIF of krypton," in Proceedings of the Combustion Institute, Vol. 33 (Combustion Institute, 2011), pp. 759-766.

7. M. Richter, Z. S. Li, and M. Alden, "Application of two-photon laser-induced fluorescence for single-shot visualization of carbon monoxide in a spark ignited engine," Appl. Spectrosc. 61, 1-5 (2007).

8. U. Westblom and M. Alden, "Laser-induced fluorescence detection of NH3 in flames with the use of 2-photon excitation," Appl. Spectrosc. 44, 881-886 (1990).

9. K. Nyholm, R. Fritzon, N. Georgiev, and M. Alden, "Twophoton induced polarization spectroscopy applied to the detection of NH3 and CO molecules in cold flows and flames," Opt. Commun. 114, 76-82 (1995).

10. J. E. M. Goldsmith, "Photonchemical effects in 2-photonexcited fluorescence detection of atomic oxygen in flames," Appl. Opt. 26, 3566-3572 (1987).

11. J. E. M. Goldsmith, "Two-photon-excited stimulatedemission from atomic-hydrogen in flames," J. Opt. Soc. Am. B 6, 1979-1985 (1989).

12. N. Georgiev, K. Nyholm, R. Fritzon, and M. Alden, "Developments of the amplified stimulated-emission technique for spatially resolved species detection in flames," Opt. Commun. 108, 71-76 (1994).

13. A. D. Tserepi, E. Wurzberg, and T. A. Miller, "Two-photonexcited stimulated emission from atomic oxygen in RF plasmas: detection and estimation of its threshold," Chem. Phys. Lett. 265, 297-302 (1997).

14. L. W. Casperson, "Rate-equation approximations in high-gain lasers," Phys. Rev. A 55, 3073-3085 (1997).

15. J. W. Daily, "Use of rate equations to describe laser excitation in flames," Appl. Opt. 16, 2322-2327 (1977).

16. J. Amorim, G. Baravian, and J. Jolly, "Laser-induced resonance fluorescence as a diagnostic technique in non-thermal equilibrium plasmas,” J. Phys. D: Appl. Phys. 33, R51-R65 (2000).

17. H. Bergstrom, H. Lundberg, and A. Persson, "Investigations of stimulated-emission on B-A lines in CO," Z. Phys. D: At. Mol. Clusters 21, 323-327 (1991).

18. Y. L. Huang and R. J. Gordon, "The effect of amplified spontaneous emission on the measurement of the multiplet state distribution of ground-state oxygen atoms," J. Chem. Phys. 97, 6363-6368 (1992).

19. J. Amorim, G. Baravian, M. Touzeau, and J. Jolly, "Twophoton laser-induced fluorescence and amplified spontaneous emission atom concentration measurements in $\mathrm{O}(2)$ and $\mathrm{H}(2)$ discharges," J. Appl. Phys. 76, 1487-1493 (1994).

20. Y. Zhao, C. N. Tong, and L. Ma, "Demonstration of a new laser diagnostic based on photodissociation spectroscopy for 
imaging mixture fraction in a non-premixed jet flame," Appl. Spectrosc. 64, 377-383 (2010).

21. Y. Zhao, C. N. Tong, and L. Ma, "Assessment of a novel flow visualization technique using photodissociation spectroscopy," Appl. Spectrosc. 63, 199-206 (2009).

22. T.B. Settersten and M. A. Linne, "Modeling pulsed excitation for gas-phase laser diagnostics," J. Opt. Soc. Am. B 19, 954-964 (2002).

23. L. Allen and G. I. Peters, "Amplified spontaneous emission and external signal amplification in an inverted medium," Phys. Rev. A 8, 2031-2047 (1973).

24. M. E. Riley, "Growth of parametric fields in $(2+1)$-photon laser ionization of atomic oxygen," Phys. Rev. A 41, 4843-4856 (1990).

25. Y. Zhao and L. Ma, "Multidimensional Monte Carlo model for two-photon LIF and amplified spontaneous emission," Comput. Phys. Commun., doi:10.1016/j.cpc.2012.02.027 (to be published).

26. M. Alden, U. Westblom, and J. E. M. Goldsmith, "Two-photonexcited stimulated emission from atomic oxygen in flames and cold gases," Opt. Lett. 14, 305-307 (1989).
27. S. Agrup, F. Ossler, and M. Alden, "Measurements of collisional quenching of hydrogen atoms in an atmosphericpressure hydrogen oxygen flame by picosecond laser-induced fluorescence," Appl. Phys. B 61, 479-487 (1995).

28. K. Niemi, V. Schulz-von der Gathen, and H. F. Dobele, "Absolute calibration of atomic density measurements by laser-induced fluorescence spectroscopy with twophoton excitation,” J. Phys. D: Appl. Phys. 34, 2330-2335 (2001).

29. L. Cerdan, A. Costela, and I. Garcia-Moreno, "On the characteristic lengths in the variable stripe length method for optical gain measurements," J. Opt. Soc. Am. B 27, 1874-1877 (2010).

30. R. C. Y. Auyeung, D. G. Cooper, S. Kim, and B. J. Feldman, "Stimulated-emission in atomic-hydrogen at $656 \mathrm{~nm}$," Opt. Commun. 79, 207-210 (1990).

31. W. L. Wiese, M. W. Smith, and B. M. Glennon, "Atomic Transition Probabilities," Vol. 1, National Standard Reference Data Series, NSRDS-NBS, Issue 4 (National Bureau of Standards, 1966). 